\title{
Liver and biliary
}

\section{Empyema of the gall bladder - reappraisal of a neglected disease}

\author{
J R THORNTON, K W HEATON, H J ESPINER, AND W K ELTRINGHAM \\ From the University Department of Medicine and Department of Surgery, Bristol Royal Infirmary, Bristol
}

SUmmary Thirty two patients with empyema of the gall bladder were identified among 1327 cases of gall-bladder disease presenting to one hospital over a six year period. Abdominal pain had been present for a median of eight days and, in eight cases, for between one and four months. In a few cases, the disease was painless and was discovered unexpectedly at postmortem or at operation for unrelated disease. The serious nature of the complaint was belied by the often scanty physical signs. Less than half the patients had a pyrexia of more than $37.5^{\circ} \mathrm{C}$ and the presence of sepsis was rarely suspected clinically. Eight patients $(25 \%)$ died, usually from unsuspected septicaemia. This considerable mortality might be reduced by the wider use of blood culture in cases of apparent 'cholecystitis' and by greater awareness that empyema of the gall bladder is sometimes chronic, painless, and afebrile.

Empyema of the gall bladder was described extensively in surgical texts of the early years of this century ${ }^{1-3}$ but now receives scant mention ${ }^{4}$ or none at all. ${ }^{56}$ In addition, it appears to have been largely forgotten that its course can be chronic. ${ }^{2} 3$ This may be because of the widespread use of antibiotics, along with the increasing adoption of a policy of early cholecystectomy for acute gall-bladder disease. $^{7-9}$ We have recently cared for several elderly women in whom the disease was a terminal, but remarkedly indolent, event. As the number of old people in the population is rising, it seemed important to determine whether this neglected disease is still an important problem and to redefine its natural history. We decided, therefore, to review all cases of empyema of the gall bladder which had presented to our hospital over a six year period.

\section{Methods}

\section{PATIENTS}

The names of all the patients treated in the Bristol Royal Infirmary for gall-bladder disease during the six years 1976 to 1981 inclusive were identified. All the patients had a diagnosis as listed on their discharge summary which fell within code 574

Address for correspondence: Dr J R Thornton, University Department of Medicine. St James Hospital, Leeds.

Received for publication 25 February 1983. (cholelithiasis) or code 575 (gall-bladder disease without mention of stones) of the International Classification of Diseases, 8th revision. One thousand, three hundred and ninety two patients were identified in this manner. The case notes of $1327(95.3 \%)$ were successfully retrieved and examined.

Empyema was defined as an inflamed gall bladder which contained pus. The presence of inflammation and pus were established from the operation record, the gall-bladder swab report, and the histology report on the resected gall bladder, or from postmortem findings. Cases of generalised infection of the biliary tree were designated as cholangitis and excluded from the study. From the 1327 sets of case notes, 32 patients $(2.4 \%)$ were identified who fulfilled these criteria and, on stringent review by all the authors, were agreed to be true examples of this disease. These cases were analysed to determine their age and sex distribution, and their clinical and pathological features. The statistical significance of differences was determined by either Student's $t$ test or Fisher's exact test, as appropriate.

\section{Results}

The 32 patients were mostly elderly, their mean age being 71 years (range 47-91 years). There were 19 women and 13 men, giving a female preponderance of 1.5 to one. 
Four patients had no abdominal pain and the empyemas were found unexpectedly, two at postmortem and the others at operations for unrelated disease. In a further two patients, empyema was found at routine cholecystectomy after a long history of apparently mild pain. The remaining 26 patients $(81 \%)$ presented with abdominal pain of sufficient severity to merit emergency admission. The pain had been present, however, to some degree, for a median of eight days and in eight patients $(25 \%)$, the pain had been present for between one and four months.

Most $(94 \%)$ of the patients were tender in the right hypochondrium. Other signs were less constant. Guarding was noted in only 18 of the 32 patients $(56 \%)$ and a palpable mass was present in only $13(41 \%)$. There was clinically recognisable jaundice in seven patients $(22 \%)$.

Despite the presence of intra-abdominal pus, only 14 patients $(44 \%)$ had a pyrexia of more than $37.5^{\circ} \mathrm{C}$ at any time preoperatively. A white cell count of more than $10 \times 10^{9} / 1$, however, was found in all but four $(88 \%)$. Liver function tests were performed in 25 of the patients and showed one or more abnormalities in $23(92 \%)$ but there was no consistent pattern of derangement. The preoperative, differential diagnosis as written in the case notes included empyema of the gall bladder in only four cases $(13 \%)$.

In all 28 patients who came to laparotomy, the procedure performed was cholecystectomy. At laparotomy (or, in four cases, necropsy) all the patients had a gall bladder obstructed by stones. In five cases $(16 \%)$ the gall bladder was perforated. A swab of the gall-bladder contents, which was available in 26 of the patients, revealed the presence of white cells in all. Aerobic culture of gall-bladder contents was positive in 21 of these cases: the remaining five patients had all been treated with antibiotics preoperatively. The most common organisms isolated were coliforms but the range of bacteria was wide, and in 10 cases there was a mixed growth of two or three bacterial species. Blood cultures were taken preoperatively in only six patients (19\%) and were positive in two cases: yielding the same organism as grown from the gall bladder.

Eight patients $(25 \%)$ died. The patients who died were older than those who survived $(83 \pm 1$ vs $67 \pm 3$ years, $p<0.001)$. They were more likely to have had little or no pain $(5 / 8$ vs $1 / 24, \mathrm{p}<0.05)$, more likely to have had an abdominal mass $(6 / 8$ vs $7 / 24, \mathrm{p}<0.05)$, and less likely to have had abdominal guarding ( $2 / 8$ vs 16/24, $\mathrm{p}<0.05)$. There were no significant differences between fatal cases and survivors in the frequency of abdominal tenderness, raised white cell count, raised temperature, gall-bladder perforation, or preoperative treatment with antibiotics. The fatalities included all four patients who did not have a cholecystectomy. The causes of death were probable or confirmed septicaemia (five cases), septicaemia and pneumonia (one), pulmonary embolus (one), and postoperative stroke (one).

\section{Discussion}

This study shows that empyema mainly affects elderly people and has a high mortality. While it usually presents with abdominal pain, the disease is sometimes painless. When pain is present, it may be tolerated for a surprisingly long time before hospital admission is arranged. Our patients had pain for a median of eight days before hospital admission and, in eight cases, pain was present for between one and four months. This indolent presentation was familiar to surgeons of the earlier part of this century, ${ }^{1-3}$ but seems largely forgotten today.

The serious nature of the disease is often belied by the scanty physical signs. In particular, less than half ${ }^{*}$ the patients had a temperature of more than $37 \cdot 5^{\circ} \mathrm{C}$. This subdued response to infection, which is perhaps related to the often advanced age of the patients, makes it difficult for the general practitioner to know whether to refer the patient to hospital and for the surgeon to recognise the need for urgent operation. The great majority of patients had abdominal tenderness, a raised white cell count and non-specific derangement of the standard liver function tests. Unfortunately, these three abnormalities are also common in cholecystitis without sepsis. Both share the common initiating event of gall-bladder obstruction by stones. Our data suggest that it is difficult or impossible to distinguish empyema from 'chemical' cholecystitis or the transition from one to the other, either clinically or biochemically. As empyema is such a dangerous condition this is, perhaps, an argument in favour of 'early' cholecystectomy in cases of acute gall-bladder disease. $^{7-9}$

When antibiotics had not been given preoperatively, culture of gall-bladder pus always grew one or more species of aerobic organisms. This finding shows that empyema of the gall bladder is rarely caused by anaerobic bacteria alone.

The true frequency of septicaemia complicating empyema is uncertain, as a blood culture was taken in less than a fifth of the patients. This illustrates that the presence of infection was often unsuspected. We suggest the wider use of blood culture in cases of apparent 'cholecystitis' so as to detect possible complicating septicaemia. This is of particular importance in the elderly in whom 
septicaemia is commonly occult, and who may be less able than younger patients to resist the infection.

Of the total group of 1327 patients, only $2.4 \%$ developed an empyema. Why sepsis supervened in these few cases remains uncertain. Of possible importance may be the age of the patients (mean: 71 years) and the length of time the gall bladder had probably been obstructed, pain having been present for a median of eight days. Some patients, however, were both relatively young and had had pain for only a day or two, so these cannot be the only predisposing factors. Gall stones become more prevalent with increasing age, ${ }^{1011}$ and with the rising number of old people in the population, empyema of the gall bladder is likely to become a more common problem.

One in four of our patients died. Some mortality may be inevitable in this disease because of the advanced age of the patients. Some deaths, however, might be avoided if blood cultures were taken in more cases of apparent 'cholecystitis' and if doctors were more aware that empyema of the gall bladder is sometimes chronic, painless, and afebrile.

We gratefully acknowledge the help of the staff of the Medical Records Department of the Bristol Royal Infirmary in extracting the case notes from the record library.

\section{References}

1 Robson AWM, Dobson JF. Diseases of the gall-bladder and bile-ducts, including gall-stones. London: Bailliere Tindall, 1904: 85.

2 Moynihan BGA. Gall-stones and their surgical treatment. Philadelphia: W B Saunders, 1905: 196.

3 Weiss S. Diseases of the liver, gall bladder, ducts and pancreas - their diagnosis and treatment. New York: Hoeber, 1935: 668.

4 Harding Rains AJ, Ritchie HD. Bailey and Love's short practice of surgery. London: H K Lewis, 1981.

5 Sabiston DC. Textbook of surgery. The biological basis of modern surgical practice. Philadelphia: W B Saunders, 1977.

6 Schwartz SI. Principles of surgery. New York: McGraw-Hill, 1979.

7 Jones PF. Acute cholecystitis: a case for early surgery? Br Med J 1982: 285: 1376-7.

8 Mitchell A, Morris PJ. Trends in management of acute cholecystitis. $\mathrm{Br}$ Med J 1982: 284: 27-30.

9 Espiner HJ. Emergency cholecystectomy: towards guaranteed safety. In: Wilson EH, Marsden AK, eds. Care of the acutely ill and injured. New York: John Wiley, 1982: 385-7.

10 Lieber MM. The incidence of gallstones and their correlation with other diseases. Ann Surg 1952: 135: 394-405.

11 Torvick A, Hoivik B. Gallstones in an autopsy series. Incidence, complications and correlations with carcinoma of the gallbladder. Acta Chir Scand 1960: 120: $168-74$. 